\title{
Enhancing Groundwater Quality through Computational Modeling and Simulation to Optimize Transport and Interaction Parameters in Porous Media
}

\author{
Akhil Waghmare', Padmanabhan Seshaiyer ${ }^{2}$ \\ ${ }^{1}$ Thomas Jefferson High School for Science and Technology, Alexandria, VA, USA \\ ${ }^{2}$ Mathematical Sciences, George Mason University, Fairfax, VA, USA \\ Email: pseshaiy@gmu.edu
}

Received 2 December 2014; accepted 24 March 2015; published 25 March 2015

Copyright (C) 2015 by authors and Scientific Research Publishing Inc.

This work is licensed under the Creative Commons Attribution International License (CC BY). http://creativecommons.org/licenses/by/4.0/

(c) (i) Open Access

\begin{abstract}
There is a shortage of high quality drinking water caused by the introduction of contaminants into aquifers from various sources including industrial processes and uncontrolled sewage. Studies have shown that colloids, collections of nanoparticles, have the potential to remediate polluted groundwater. For such applications of nanoparticles, it is important to understand the movement of colloids. This study aims to enhance the previously developed MNM1D (Micro- and Nanoparticle transport Model in porous media in one-dimensional geometry) by making more realistic assumptions about physical properties of the groundwater-porous medium system by accounting for a non-constant flow velocity and the presence of electromagnetic interactions. This was accomplished by coupling the original model with the Darcy-Forchheimer fluid model, which is specific to transport in porous media, coupled with electromagnetic effects. The resulting model also accounts for attachment and detachment phenomena, both of the linear and Langmuirian type, as well as changes to hydrochemical parameters such as maximum colloidal particle concentration in the porous medium. The system of partial-differential equations that make up the model was solved using an implicit finite-difference discretization along with the iterative Newton's method. A parameter estimation study was also conducted to quantify parameters of interest. This more realistic model of colloid transport in porous media will contribute to the production of a more efficient method to counteract contaminants in groundwater and ultimately increase availability of clean drinking water.
\end{abstract}

\section{Keywords}

Water Quality, Computational Modeling, Colloid Transport 


\section{Introduction}

According to a 2013 report of the World Health Organization ([1] and references therein), there are over 780 million people without access to clean drinking water. Over $50 \%$ of the United States population receives its drinking water from groundwater, water that flows underneath the earth's surface in aquifers [2]. Largely due to the recent surge in industrial processes, contaminants have been introduced into groundwater, reducing the amount that can be used for drinking and requiring more expensive treatment processes. Recent incidents of water contamination [3] include radionucleotides leaking from reactors in Fukushima, Japan; petroleum spilling from crude oil pipeline in Yellowstone River, Montana; and a benzene spill in Songhua, China. Studies in the past decade also have shown that colloids, collections of nanoparticles, can destroy these contaminants through chemical reactions. One example is zero-valent iron nanoparticles, which use oxidation-reduction reactions to neutralize toxic metal ions [4]. It is thus important to understand the movements of colloidal particles in the system defined by the groundwater and porous medium that it flows through. In the past couple decades, a few models have been introduced to describe this system. An extensive presentation of the evolution of the current state of surface water and ocean water contamination models can be found in Bahadur et al. One can also find suitable mathematical models for groundwater flow in [5] and parameter identification models in [6]. A large number of suite of codes have been developed by U.S. Salinity Laboratory (USSL) and the University of California, Riverside (UCR) in the last three decades. Simunek et al. describe the historical development and applications of the HYDRUS and STANMOD software packages and related codes.

The STANMOD suite builds off analytic models developed from 1980, including CFITM and CXTFIT, which accounts for particle degradation and the equilibrium state at a single reaction site. The numerical HYDRUS model released in 1991 includes temperature dependent kinetic coefficients and allows for two reaction sites [7]. One of the models MNM1D [8], which is introduced in the last few years, introduces non-linear reaction sites and kinetic coefficients dependent upon salt concentrations. It is based on the following major components of the system: the diffusion of the nanoparticles in the fluid and the transport of them between the liquid and solid phases through reaction sites. Other features of the model include variable hydrochemical parameters that depend on the diffusion of salts and reaction sites of both the linear and Langmuirian type. This model was tested by Tosco and Sethi against both the STANMOD and HYDRUS-1D models, and the MNM1D closely matched these.

Although the existing model encompasses the previous mentioned properties, there are still additional improvements that can be made. In this study, the flow velocity of the fluid was assumed to be non-constant, governed by the Darcy-Forchheimer fluid model. The electromagnetic interactions that occur in the fluid were also introduced by adding an electric field term based on the Helmholtz-Smoluchowski equation to the mentioned fluid model. In addition, a parameter estimation algorithm was implemented for a single kinetic coefficient. These parameters govern the ionic strength of the surrounding groundwater, potentially giving researchers the ability to attribute certain characteristics to the liquid.

The structure of the paper is as follows. Section 2 introduces the mathematical model and governing differential equations. Section 3 describes the implementation of the governing system via implicit finite-difference discretization. In Section 4, we present computational experiments for a benchmark application that involves step-wise injection of nanoparticles. This application, which was presented in [9], was successfully validated. The application was enhanced and implemented with the inclusion of the non-constant flow model and electromagnetic effects. Experimental data was simulated to quantify one of the kinetic transport coefficients in order to identify the ionic strength of the groundwater. The computational framework along with the results provided in this paper suggests that the enhanced model presented is a reliable candidate for evaluating potential realworld solutions to contaminated aquifers.

\section{Mathematical Models and Background}

Transport of colloids is governed by advection-dispersion phenomena and particle-soil interactions. Colloidal particle transport in porous systems is modeled using a modified advection-dispersion equation. It describes the dual-phase, non-equilibrium interactions between particles in the liquid and solid phase. Colloid deposition on the grain surface is generally referred to as attachment, colloid release from the surface as detachment. Earlier theories, such as the classical filtration theory (CFT) consider first-order attachment kinetics, but consider the detachment ones to be negligible. However, MNM1D includes both phenomena in the particle-soil interactions. 
The physical system described is summarized in Figure 1 below.

Several 1D forms of the model have been proposed, each taking into account some or all of the above characteristics. However, we can write the general form of the modified advection-dispersion equation for the colloid movement taking into account the liquid-solid phase transfer:

$$
n \frac{\partial c}{\partial t}+\rho_{b} \frac{\partial s_{1}}{\partial t}+\rho_{b} \frac{\partial s_{2}}{\partial t}=n D \frac{\partial^{2} c}{\partial x^{2}}-\frac{\partial(v c)}{\partial x}
$$

where:

$c=$ The colloid number concentration in the liquid phase.

$s_{1}=$ The number colloid concentration in the solid phase at the first reaction site.

$s_{2}=$ The number colloid concentration in the solid phase at the second reaction site.

$\rho_{b}=$ The bulk density of the solid matrix.

$D=$ The hydrodynamic dispersion coefficient.

$v=$ The Darcyan flow velocity.

$n=$ The porosity of the packed bed.

Many types of reaction sites exist, and thus there are many models that describe the mass transfer between the solid and liquid phases. MNM1D takes into account two reaction sites. The first is assumed to have blocking phenomena, and so the colloid concentration at the site is limited to a fixed values $s_{\max }$. The transfer can be described by:

$$
\rho_{b} \frac{\partial s_{1}}{\partial t}=n\left(1-\frac{s_{1}}{s_{\max }}\right) k_{a, 1} c-\rho_{b} k_{d, 1} s_{1}
$$

where:

$k_{a, 1}=$ The attachment coefficient at site 1 .

$k_{d, 1}=$ The detachment coefficient at site 1 .

$s_{\max }=$ The maximum particle concentration for site 1 .

The second site is assumed to have a linear exchange term, and so the transfer can be described as:

$$
\rho_{b} \frac{\partial s_{2}}{\partial t}=n k_{a, 2} c-\rho_{b} k_{d, 2} s_{2}
$$

where:

$k_{a, 2}=$ The attachment coefficient at site 2 .

$k_{d, 2}=$ The detachment coefficient at site 2 .

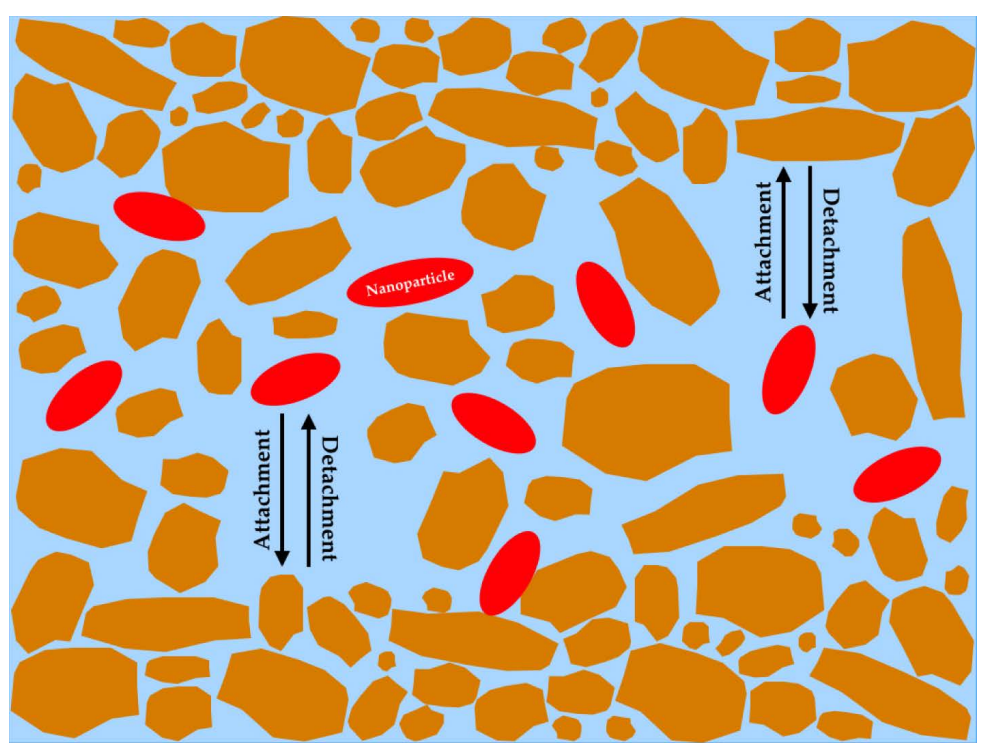

Figure 1. Groundwater system. 
The hydrochemical parameters, such as ionic strength, can be correlated with the concentration of some conservative tracer, such as salt. The transport of the tracer can also be described by the advection-dispersion equation as:

$$
n \frac{\partial c_{t}}{\partial t}=n D \frac{\partial^{2} c_{t}}{\partial x^{2}}-\frac{\partial\left(v c_{t}\right)}{\partial x}
$$

where:

$c_{t}=$ The conservative salt concentration.

The concentration of the tracer ultimately affects the values of the attachment/detachment kinetic coefficients. The functional relationship between the salt concentration and kinetic coefficients below was proposed in [8].

$$
\begin{gathered}
k_{a, i}=\frac{k_{a \infty, i}}{1+\left(\frac{\mathrm{CDC}_{i}}{c_{t}}\right)^{\beta_{a, i}}} \\
k_{d, i}=\frac{k_{d 0, i}}{1+\left(\frac{c_{t}}{\mathrm{CRC}_{i}}\right)^{\beta_{d, i}}} \\
s_{\max }=\gamma_{s}\left(c_{t}\right)^{\beta_{s}}
\end{gathered}
$$

where:

$k_{a \infty, i}=$ Asymptotic value of $k_{a, i}$.

$\mathrm{CDC}_{i}=$ Critical deposition concentration.

$\mathrm{CRC}_{i}=$ Critical release concentration.

$k_{d 0, i}=$ Asymptotic value of $k_{d, i}$.

$\gamma_{s}, \beta_{a, i}, \beta_{d, i}, \quad \beta_{s}=$ Empirical coefficients determined by breakthrough curves of kinetic coefficients.

Finally, we now introduce a fluid model to describe the flow velocity of the groundwater over time and space. Here, we will implement the Darcy-Forchheimer model [10], which has specific applications to groundwater flow. The model is governed by the following differential equations:

$$
\begin{gathered}
\frac{\mathrm{d}^{2} p}{\mathrm{~d} x^{2}}=0 \\
-\frac{\mathrm{d} p}{\mathrm{~d} x}=\frac{\mu}{k} v+\beta \rho v^{2}
\end{gathered}
$$

where:

$p=$ Pressure of the fluid.

$\mu=$ Viscosity of the fluid.

$k=$ Permeability of the fluid.

$\beta=$ Inertial factor of the fluid.

$\rho=$ Density of the fluid.

We now wish to introduce how the presence of electromagnetics affects this model. To do this, we will turn to a modified version of the above fluid model goverened by the Helmholtz-Smoluchowski (H-S) equation as discussed in [11]. The H-S equation relates the electric field present in the groundwater with physical characteristics of the system:

$$
\mathbf{E}=\frac{\epsilon_{f} \zeta}{\mu \sigma_{f}} \nabla p
$$

where:

$\mathbf{E}=$ Electric field.

$\epsilon_{f}=$ Dielectric permittivity of fluid.

$\zeta=$ Zeta potential. 
$\sigma_{f}=$ Fluid conductivity.

As described by Fourie, the fluid model then becomes:

$$
\begin{gathered}
\frac{\mathrm{d}^{2} p}{\mathrm{~d} x^{2}}=0 \\
-\frac{\mathrm{d} p}{\mathrm{~d} x}=\frac{1}{\phi} v+\frac{k \beta \rho}{\mu \phi} v^{2} \\
\phi=\frac{k}{\mu}-\frac{L \epsilon_{f} \zeta}{\mu \sigma_{f}}
\end{gathered}
$$

where:

$L=$ Electrokinetic coupling coefficient.

\section{Implicit Finite Difference Solution Methodology}

In this section, we will develop an implicit finite difference scheme to solve the coupled system Equations (5)(14). To rewrite the derivatives of $c(x, t), c_{t}(x, t), s_{1}(x, t)$, and $s_{2}(x, t)$, we first consider the following finite difference approximations for some function $f(x, t)$ expressed as:

$$
\begin{gathered}
\frac{\partial f}{\partial t} \approx \frac{f_{i, n+1}-f_{i, n}}{\Delta t} \\
\frac{\partial f}{\partial x} \approx \frac{f_{i+1, n+1}-f_{i-1, n+1}}{2 \Delta x} \\
\frac{\partial^{2} f}{\partial x^{2}} \approx \frac{f_{i+1, n+1}-2 f_{i, n+1}+f_{i-1, n+1}}{(\Delta x)^{2}}
\end{gathered}
$$

where $f_{i, n}=f((\Delta x) i,(\Delta x) n)$. In order to solve this system, we first need values of $v_{i-1, n+1}, v_{i, n+1}$, and $v_{i+1, n+1}$. We can extract these values from Equations (8)-(10). Using a central difference approximation, we can write Equation (8) as:

$$
\frac{p_{i+1, n+1}-2 p_{i, n+1}+p_{i-1, n+1}}{(\Delta x)^{2}}=0
$$

which implies

$$
p_{i, n+1}=\frac{1}{2}\left(p_{i+1, n+1}+p_{i-1, n+1}\right)
$$

We now see that in order to solve for pressure, we require initial values for $p$ at $t=0$ for all interested positions, and that this equation is independent of the rest of the system. We now rewrite Equation (9) as:

$$
-\frac{p_{i+1, n+1}-p_{i-1, n+1}}{2 \Delta x}=\frac{1}{\phi} v_{i, n}+\frac{k \beta \rho}{\mu \phi} v_{i, n}^{2}
$$

We can solve for $v_{i, n}$ using the quadratic formula, and so we have

$$
v_{i, n}=\frac{-\frac{1}{\phi}+\sqrt{\frac{1}{\phi^{2}}-4\left(\frac{k \beta \rho}{\mu \phi}\right)\left(\frac{p_{i+1, n+1}-p_{i-1, n+1}}{2 \Delta x}\right)}}{\frac{2 k \beta \rho}{\mu \phi}}
$$

We see here that $v_{i, n}$ also appears to be independent of our interested quantities. However, to make the model more realistic, one could assume that $\rho$ is a function of the local concentration of the nanoparticles, $\rho=h\left(c_{i, n}\right)$.

Note, for the sake of simplicity, we will assume $k_{a, i}, k_{d, i}$, and $s_{\max , 1}$ to be constant. The values will be 
determined by those used in Tosco and Sethi. Using the approximations, Equations (1)-(4) can be rewritten as:

$$
\begin{gathered}
n\left(\frac{c_{t_{i, n+1}}-c_{t_{i, n}}}{\Delta t}\right)=n D\left(\frac{c_{t_{i+1, n+1}}-2 c_{t_{i, n+1}}+c_{t_{i-1, n+1}}}{(\Delta x)^{2}}\right)-v_{i, n+1}\left(\frac{c_{t_{i+1, n+1}}-c_{t_{i-1, n+1}}}{2 \Delta x}\right)-c_{t_{i, n+1}}\left(\frac{v_{i+1, n+1}-v_{i-1, n+1}}{2 \Delta x}\right) \\
n\left(\frac{c_{i, n+1}-c_{i, n}}{\Delta t}\right)+\rho_{b}\left(\frac{s_{1_{i, n+1}}-s_{1_{i, n}}}{\Delta t}\right)+\rho_{b}\left(\frac{s_{2_{i, n+1}}-s_{2_{i, n}}}{\Delta t}\right) \\
=n D\left(\frac{c_{i+1, n+1}-2 c_{i, n+1}+c_{i-1, n+1}}{(\Delta x)^{2}}\right)-v_{i, n+1}\left(\frac{c_{i+1, n+1}-c_{i-1, n+1}}{2 \Delta x}\right)-c_{i, n+1}\left(\frac{v_{i+1, n+1}-v_{i-1, n+1}}{2 \Delta x}\right) \\
\rho_{b} \frac{s_{1_{i, n+1}}-s_{1_{i, n}}}{\Delta t}=n k_{a, 1} c_{i, n+1}-\frac{n k_{a, 1}}{s_{\max }} s_{1_{i, n+1}} c_{i, n+1}-\rho_{b} k_{d, 1} S_{1_{i, n+a}} \\
\rho_{b} \frac{s_{2_{i, n+1}}-s_{2_{i, n}}}{\Delta t}=n k_{a, 2} c_{i, n+1}-\rho_{b} k_{d, 2} s_{2_{i, n+1}}
\end{gathered}
$$

We can now solve for our interested quantities, $c_{t_{i, n}}$ and $c_{i, n}$ from the previous equations to yield

$$
\begin{gathered}
c_{t_{i, n}}=\left(1+2 \beta+\gamma\left(v_{i+1, n+1}-v_{i-1, n+1}\right)\right) c_{t_{i, n+1}}+\left(\gamma v_{i, n+1}-\beta\right) c_{t_{i+1, n+1}}-\left(\beta+\gamma v_{i, n+1}\right) c_{t_{i-1, n+1}} \\
c_{i, n}=\left(1+2 \beta+\gamma\left(v_{i+1, n+1}-v_{i-1, n+1}\right)\right) c_{i, n+1}+\left(\gamma v_{i, n+1}-\beta\right) c_{i+1, n+1}-\left(\beta+\gamma v_{i, n+1}\right) c_{i-1, n+1}+\alpha\left(s_{1_{i, n+1}}+s_{2_{i, n+1}}-s_{1_{i, n}}-s_{2_{i, n}}\right)
\end{gathered}
$$

and by a similar method, we can solve for $s_{1_{i, n}}$ and $s_{2_{i, n}}$ to get

$$
\begin{gathered}
S_{1_{i, n}}=\omega_{1} S_{1_{i, n+1}}+\left(\frac{\tau_{1} S_{1_{i, n+1}}}{S_{\max }}-\tau_{1}\right) c_{i, n+1} \\
S_{2_{i, n}}=\omega_{2} S_{2_{i, n+1}}-\tau_{2} C_{i, n+1}
\end{gathered}
$$

where $\alpha=\frac{\rho_{b}}{n}, \quad \beta=\frac{D \Delta t}{(\Delta x)^{2}}, \gamma=\frac{\Delta t}{2 n \Delta x}, \omega_{1}=1+\Delta t k_{d, 1}, \quad \tau_{1}=\frac{n k_{a, 1} \Delta t}{\rho_{b}}, \omega_{2}=1+\Delta t k_{d, 2}$, and $\tau_{2}=\frac{n k_{a, 2} \Delta t}{\rho_{b}}$. We can write Equations (11)-(14) in matrix form as

$$
\boldsymbol{A} \boldsymbol{x}_{n}=\boldsymbol{B} \boldsymbol{x}_{n+1}
$$

where

$$
\begin{aligned}
& \boldsymbol{A}=\left[\begin{array}{llll}
C & O & O & O \\
O & C & D & D \\
O & O & C & O \\
O & O & O & C
\end{array}\right], \quad \boldsymbol{B}=\left[\begin{array}{cccc}
E & O & O & O \\
O & E & D & D \\
O & G & H & O \\
O & I & O & K
\end{array}\right] \\
& \boldsymbol{C}=\left[\begin{array}{lll}
1 & 0 & 0 \\
0 & 1 & 0 \\
0 & 0 & 1
\end{array}\right], \quad \boldsymbol{D}=\left[\begin{array}{lll}
\alpha & 0 & 0 \\
0 & \alpha & 0 \\
0 & 0 & \alpha
\end{array}\right], \quad \boldsymbol{E}=\left[\begin{array}{lll}
a & b & 0 \\
c & a & b \\
0 & c & a
\end{array}\right] \\
& \boldsymbol{G}=\left[\begin{array}{ccc}
\left(d s_{1_{1}}^{n+1}-\tau_{1}\right) & 0 & 0 \\
0 & \left(d s_{1_{2}}^{n+1}-\tau_{1}\right) & 0 \\
0 & \left(d s_{1_{3}}^{n+1}-\tau_{1}\right) & 0
\end{array}\right], \quad \boldsymbol{H}=\left[\begin{array}{ccc}
\omega_{1} & 0 & 0 \\
0 & \omega_{1} & 0 \\
0 & 0 & \omega_{1}
\end{array}\right] \\
& \boldsymbol{I}=\left[\begin{array}{ccc}
-\tau_{2} & 0 & 0 \\
0 & -\tau_{2} & 0 \\
0 & 0 & -\tau_{2}
\end{array}\right], \quad \boldsymbol{K}=\left[\begin{array}{ccc}
\omega_{2} & 0 & 0 \\
0 & \omega_{2} & 0 \\
0 & 0 & \omega_{2}
\end{array}\right], \quad \boldsymbol{O}=\left[\begin{array}{lll}
0 & 0 & 0 \\
0 & 0 & 0 \\
0 & 0 & 0
\end{array}\right]
\end{aligned}
$$




$$
x_{n}=\left[\begin{array}{llllllllllll}
c_{t_{1}}^{n} & c_{t_{2}}^{n} & \cdots & c_{1}^{n} & c_{2}^{n} & \cdots & s_{1_{1}}^{n} & s_{1_{2}}^{n} & \cdots & s_{2_{1}}^{n} & s_{2_{2}}^{n} & \cdots
\end{array}\right]^{\mathrm{T}}
$$

With $a=1+2 \beta, \quad b=\gamma-\beta, \quad c=-(\gamma+\beta)$, and $d=\frac{\tau_{1}}{s_{\max }}$. Letting $\boldsymbol{x}_{n+1}=\mathbf{z}$, Equation (15) can be rewritten as

$$
\boldsymbol{F}(\mathbf{z})=0
$$

Due to the non-linearity that exists in matrix $\boldsymbol{B}$, Equation (16) is a non-linear system. We will use Newton's Method to approximate the solution to this non-linear system. If $\mathbf{z}_{m}$ is the $m$ th iteration of the solution vector $\mathbf{z}$, then

$$
\mathbf{z}_{m+1}=\mathbf{z}_{m}-\boldsymbol{J}^{-1}\left(\mathbf{z}_{m}\right) \boldsymbol{F}\left(\mathbf{z}_{m}\right)
$$

where the Jacobian $\boldsymbol{J}$ and $\boldsymbol{F}$ are defined as:

$$
\begin{gathered}
\boldsymbol{F}=\boldsymbol{B} \boldsymbol{z}_{m}-\boldsymbol{A} \boldsymbol{x}_{n} \\
\boldsymbol{J}=\left[\begin{array}{cccc}
\boldsymbol{E} & \boldsymbol{O} & \boldsymbol{O} & \boldsymbol{O} \\
\boldsymbol{O} & \boldsymbol{E} & \boldsymbol{D} & \boldsymbol{D} \\
\boldsymbol{O} & \boldsymbol{G} & \boldsymbol{L} & \boldsymbol{O} \\
\boldsymbol{O} & \boldsymbol{I} & \boldsymbol{O} & \boldsymbol{K}
\end{array}\right] \\
\boldsymbol{L}=\left[\begin{array}{ccc}
\left(d c_{1}^{n+1}+\omega_{1}\right) & 0 & 0 \\
0 & \left(d c_{2}^{n+1}+\omega_{1}\right) & 0 \\
0 & 0 & \left(d c_{3}^{n+1}+\omega_{1}\right)
\end{array}\right]
\end{gathered}
$$

Equation (17) can be rewritten as:

$$
\Delta \mathbf{z}_{m}=-\boldsymbol{J}^{-1}\left(\mathbf{z}_{m}\right) \boldsymbol{F}\left(\mathbf{z}_{m}\right)
$$

The solution is now obtained iteratively by calculating the residuals $\Delta \boldsymbol{z}_{m}$ and updating the solution so that $\mathbf{z}_{m+1}=\mathbf{z}_{m}+\Delta \mathbf{z}_{m}$ at each node within the computational domain. This process is repeated until the difference between the successive solution is within a prescribed tolerance. The numerical method was validated to be quadratically convergent.

\section{Computational Experiments}

In this section, we perform the following computational experiment which will be used to validate the results from [9] to the one presented in this study. We will consider a line segment from $x=0$ m to $x=10^{-1} \mathrm{~m}$, with a step-wise injection of nanoparticles at $x=0 \mathrm{~m}$ for the first $200 \mathrm{~s}$. The values of the relevant constants, which were retrieved from [12]-[16], used are listed in Table 1 below.

\subsection{Comparison at $x=1 \times 10^{-3} \mathrm{~m}$ and $x=9 \times 10^{-3} \mathrm{~m}$}

We will first compare the models at a point that is close to the source of the nanoparticles, $x=1 \times 10^{-3} \mathrm{~m}$. The bold graphs in Figure 2 and Figure 3 represent the exact results from [9]. As we can see from the graphs below, the shape of the graphs is slightly different. This change can be attributed to the fact that the flow velocity now depends on density, which now depends on the local concentration of the nanoparticles. So, as the concentration increases, the velocity will decrease, which explains why the concentration grows slower as time passes in the new model. A similar process can be used to explain why the graph decreases slower as well.

We will now compare the models at a point that is farther away from the nanoparticle source, $x=9 \times 10^{-3} \mathrm{~m}$. From the graphs below we see that there is a much larger discrepancy between the two models. One major reason for this is the fact that the concentration at this point is not very influenced by the source. Furthermore, any discrepancy from points closer to the source are now exemplified farther down. This situation thus reveals the true effect of the electromagnetic interactions that occur. 
Table 1. Values of model parameters.

\begin{tabular}{ccc}
\hline Variable & Value \\
\hline$\rho_{b}$ & $1.5 \times 10^{-6}$ \\
$D$ & 0.4 \\
$k_{a, 1}$ & $1 \times 10^{-7}$ \\
$k_{a, 2}$ & $4.95 \times 10^{-4}$ \\
$k_{d, 1}$ & $1.17 \times 10^{-5}$ \\
$k_{d, 2}$ & $4.48 \times 10^{-4}$ \\
$S_{\max }$ & $1.06 \times 10^{-3}$ \\
$\mu$ & $3.84 \times 10^{-8}$ \\
$k$ & $8.9 \times 10^{-4}$ \\
$\beta$ & 60 \\
$L$ & $1 \times 10^{9}$ \\
$\epsilon_{f}$ & $102.3 \times 10^{-6}$ \\
$\zeta$ & 78 \\
$\sigma_{f}$ & $41.7 \times 10^{-6}$ \\
$\rho$ & 0.5 \\
\hline
\end{tabular}

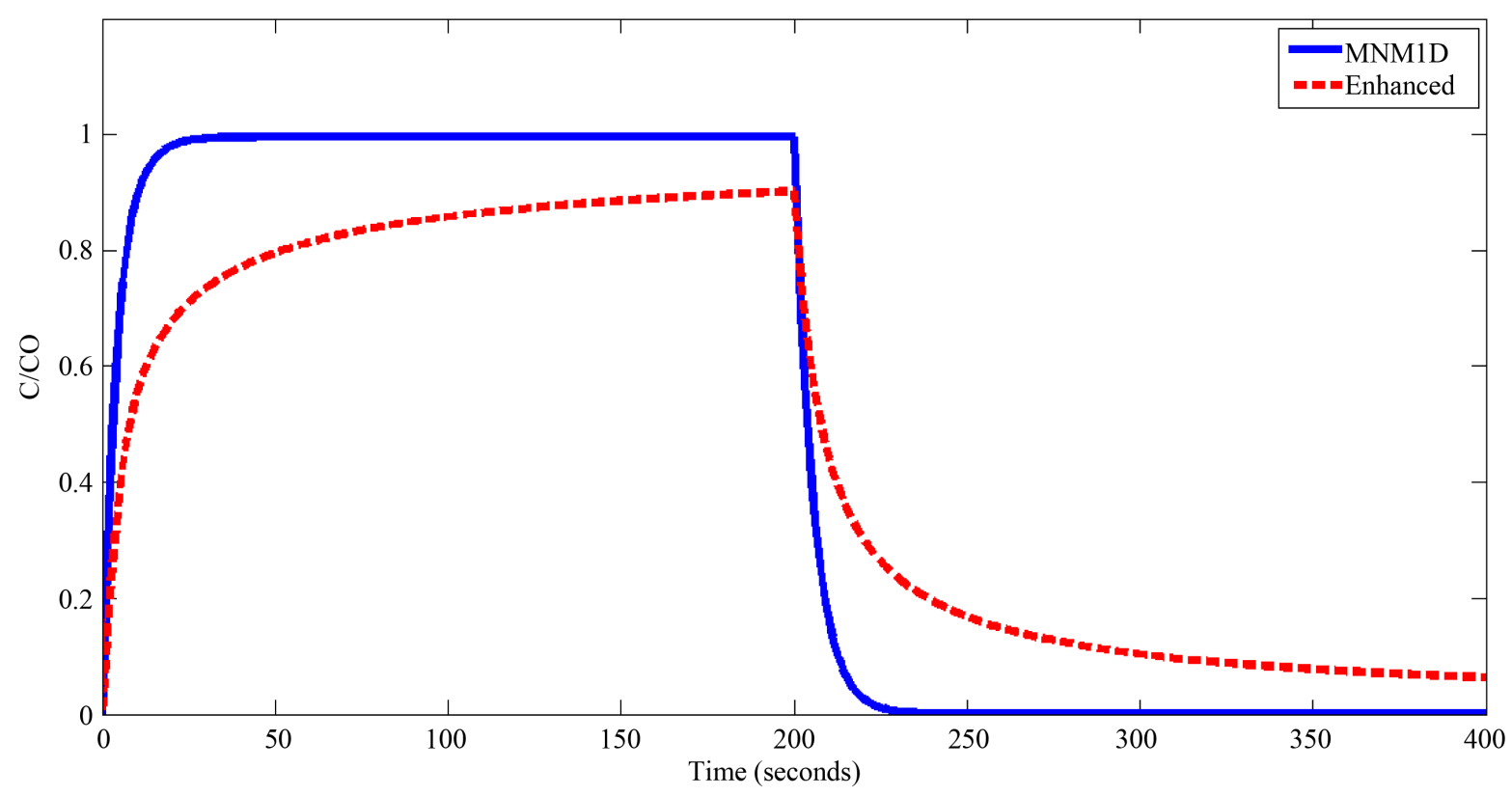

Figure 2. Comparison of simulations for MNM1D of colloid transport at $1 \mathrm{~mm}$ ionic strength at $\quad x=1 \times 10^{-3} \mathrm{~m}$.

\subsection{Graphical User Interface}

To permit users to easily test the model, a graphical user interface (GUI) was created. This allows users to enter model parameters including physical characteristics of the system, affecting both the dispersion of the colloids and the electric field, and the particle-soil interaction kinetic coefficients. The GUI presents the colloidal concentrations at both of the above positions, $x=1 \times 10^{-3} \mathrm{~m}$ and $x=9 \times 10^{-3} \mathrm{~m}$. Figure 4 and Figure 5 below are screenshots of the program at startup, one with default parameters mirroring those used in this study and another 


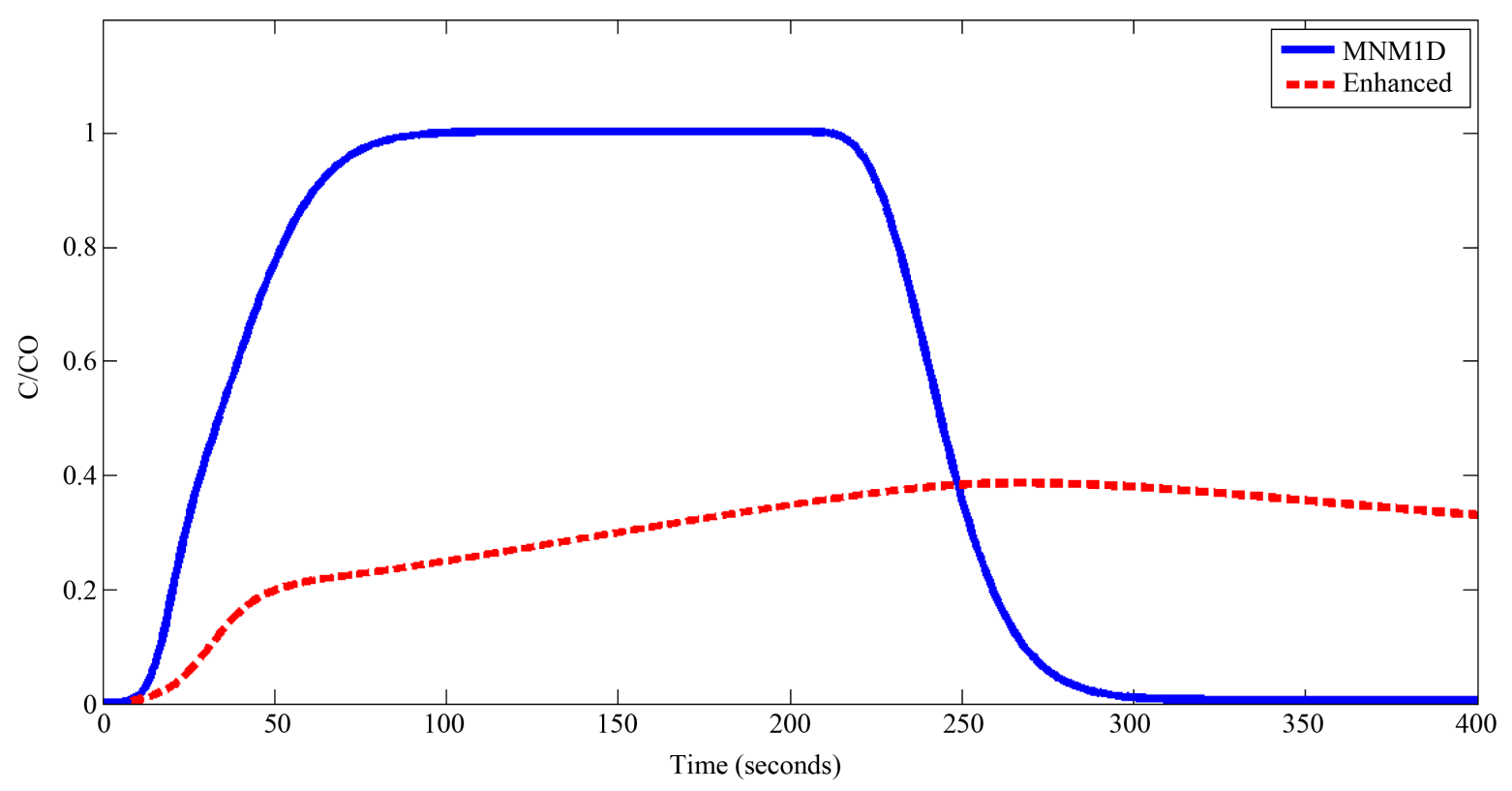

Figure 3. Comparison of Simulations for MNM1D of Colloid Transport at $1 \mathrm{mM}$ Ionic Strength at $x=9 \times 10^{-3} \mathrm{~m}$.

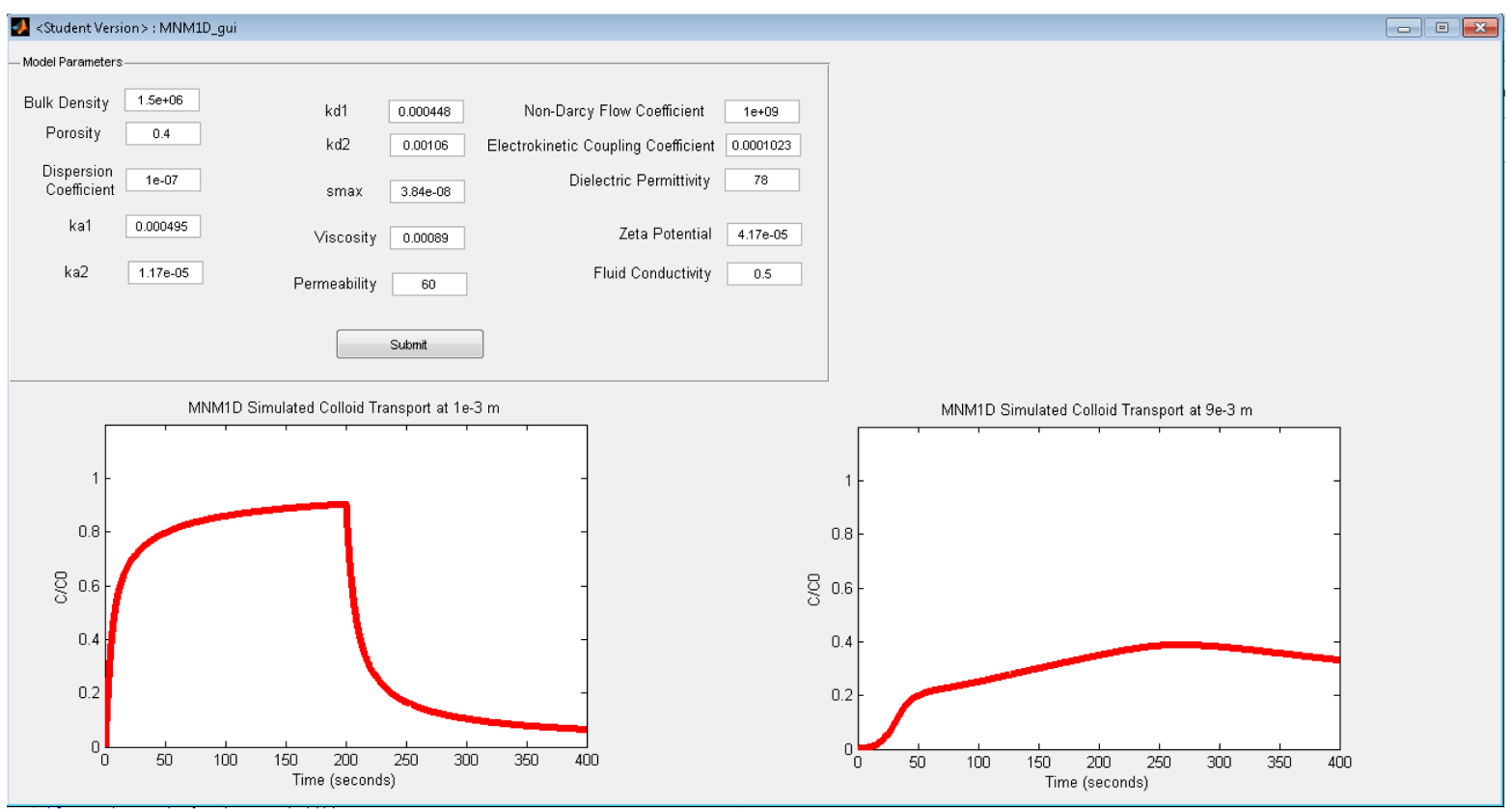

Figure 4. Graphical user interface at startup.

with user-entered parameters.

\subsection{Parameter Estimation}

The kinetic coefficients in the description of the attachment and detachment phenomena determine the ionic strength of the colloids. Being able to estimate the values of these given experimental data would allow for approximating this characteristic of the groundwater that would ultimately help determine properties such as the mobility of radioactive isotopes in the system [17]. Thus, a parameter estimation algorithm was implemented for one of these parameters $k_{a}$. To test how accurate the estimation process was, some "noise” or random deviations, were added to the computed values of concentration outputed by the model to make it more rea- 
listically represent experimental data. The accuracy and error were recorded for different magnitudes of noise, the results are shown in Figure 6 and Figure 7.

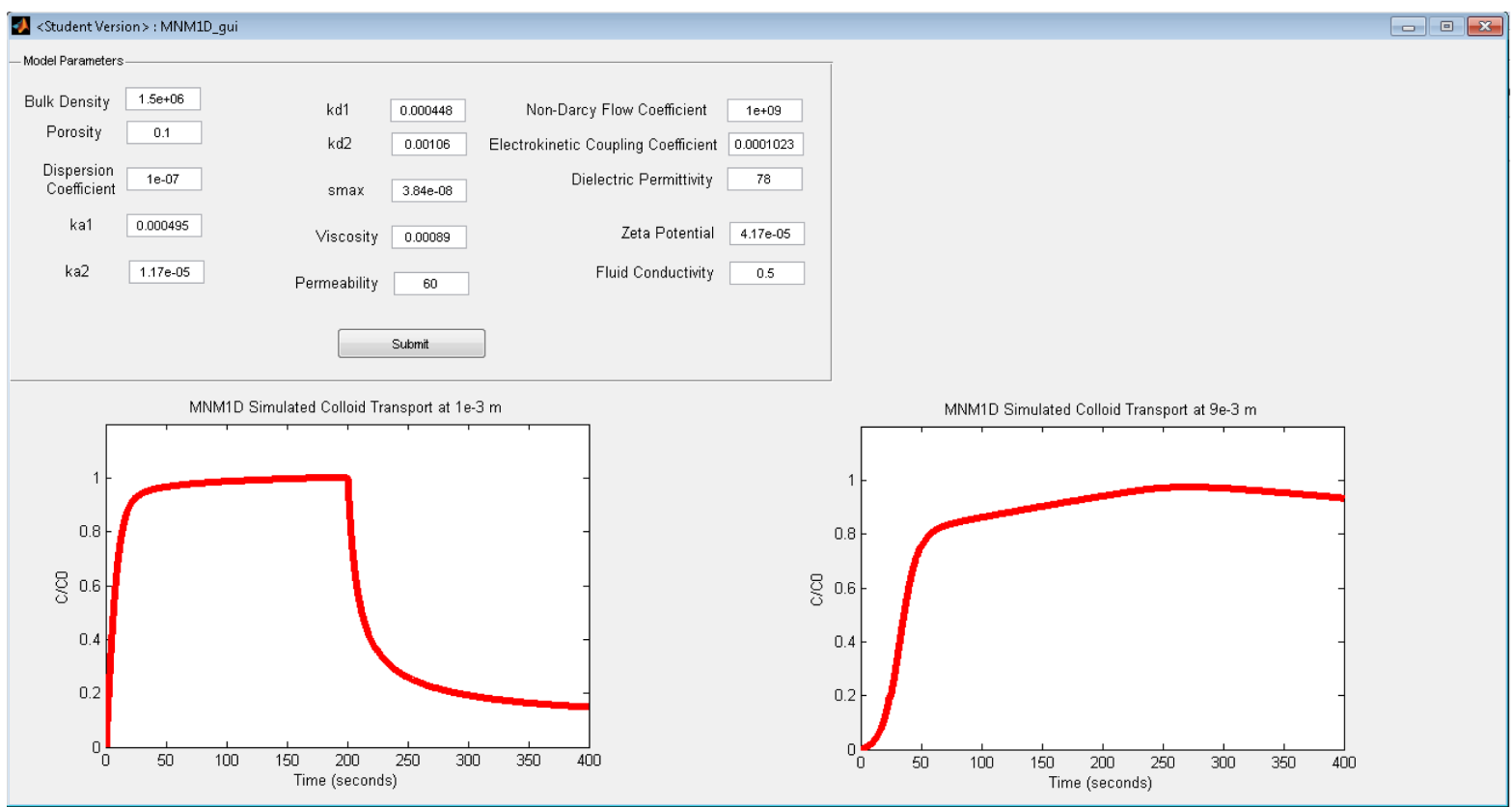

Figure 5. Graphical user interface with user parameters.

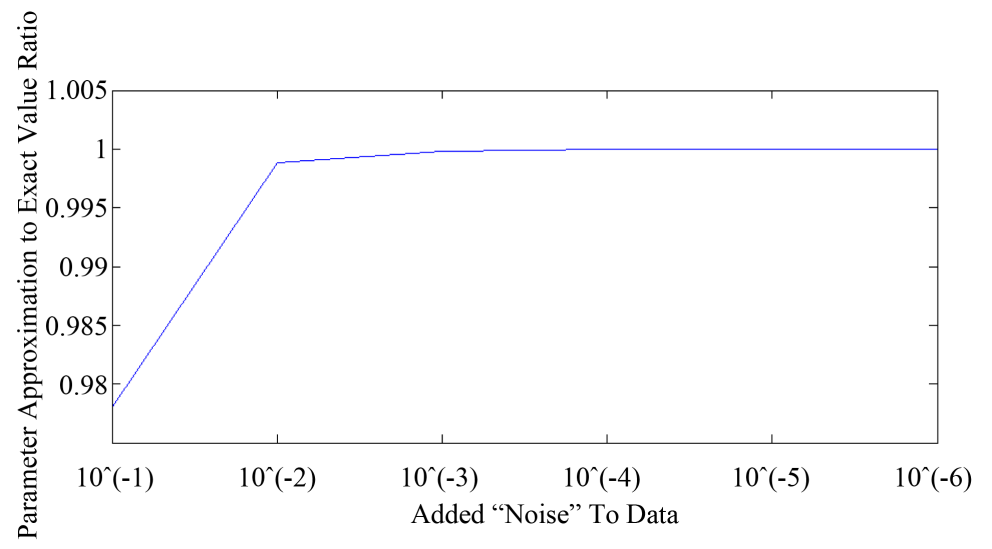

Figure 6. Parameter estimation $k_{a}$ values error.

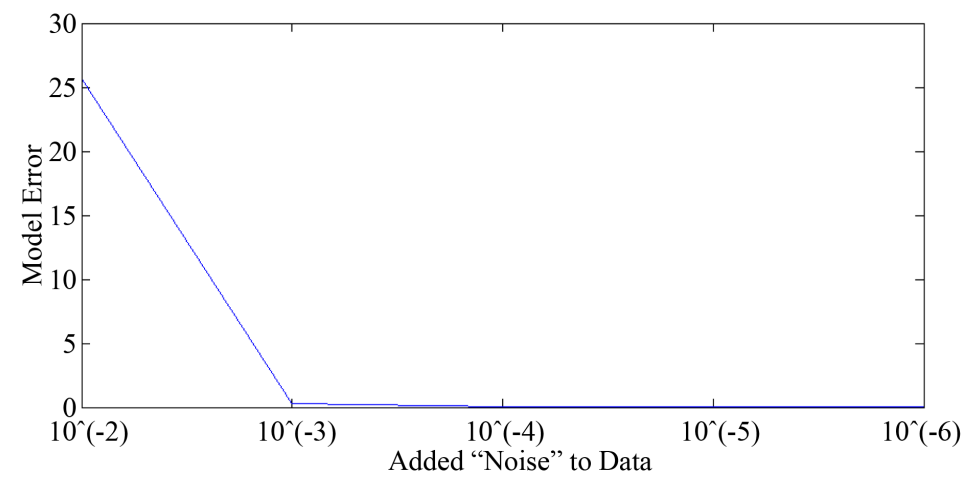

Figure 7. Parameter estimation model error. 


\section{Conclusions and Future Work}

In this work, a new enhanced model that incorporates non-constant flow velocity governed by the Darcy-Forchheimer fluid model as well as influence of electromagnetic interactions using H-S equation has been studied. We were not only able to validate the results in the absence of these effects in comparsion to published work, but were also able to show that these non-constant flow velocity and electromagnetic effects impact the colloid concentrations. Two other contributions include the development of a parameter estimation strategy to extract parameters of interest by comparing the computed data against experimental data. In this paper, the experimental data was generated by adding different levels of "noise" to the computed data. Finally, a graphical user interface, that helps users to enter model parameters and obtain the colloidal concentrations in real-time, was also presented. The software that has been developed through this work provides flexible infrastructure to evaluate the influence of parameters such as $n$ and $k_{a}$ on the nanoparticle cocentration. The GUI allows users to evaluate this influence. While we have used a finite difference algorithm, we hope to establish the stability of the method in a forthcoming paper. We also hope to study stochastic effects that may be created by the randomness of the parameters in the model and finally extend our current model to higher dimensions.

The current paper does not include explicitly the interaction of colloidal particles with various contaminants that can exist in groundwater. In a forthcoming paper, we wish to enhance our model by including this interaction as colloidal particles are well-known to remediate contaminants because of their unique physiochemical properties. Understanding this interaction will also help us to investigate how the colloidal particles can be used in the removal of various groundwater contaminants including chlrorinated compounds, pesticides, heavy metals, nitrates, and explosives in water ([18] and references therein). This will help us to enhance our software that can be more widely used by various stakeholders.

\section{References}

[1] World Health Organization (2013) Progress on Sanitation and Drinking-Water: 2013 Update, Uniform Resource Locator. WHO Press, Geneva. http://www.unicef.org/wash/files/JMP2013final_en.pdf

[2] The Groundwater Foundation (n.d.) Potential Threats to Our Groundwater. http://www.groundwater.org/get-informed/groundwater/contamination.html

[3] Bahadur, R., Amstutz, D.E. and Samuels, W.B. (2013) Water Contamination Modeling-A Review of the State of the Science. Journal of Water Resource and Protection, 5, 142-155. http://dx.doi.org/10.4236/jwarp.2013.52016

[4] Li, X.-Q., Elliot, D.W. and Zhang, W.-X. (2006) Zero-Valent Iron Nanoparticles for Abatement of Environmental Pollutants: Materials and Engineering Aspects. Critical Reviews in Solid State and Materials Sciences, 31, 111-122. http://dx.doi.org/10.1080/10408430601057611

[5] Igboekwe, M.U. and Achi, N.J. (2011) Finite Difference Method of Modelling Groundwater Flow. Journal of Water Resource and Protection, 3, 192-198. http://dx.doi.org/10.4236/jwarp.2011.33025

[6] Shao, D.G., Yang, H.D. andLiu, B.Y. (2013) Identification of Water Quality Model Parameter Based on Finite Difference and Monte Carlo. Journal of Water Resource and Protection, 5, 1165-1169. http://dx.doi.org/10.4236/jwarp.2013.512123

[7] Simunek, J., van Genuchten, M.T. and Sejna, M. (2008) Development and Applications of the HYDRUS and STANMOD Software Packages and Related Codes. Vadose Zone Journal, 7, 587-600. http://dx.doi.org/10.2136/vzj2007.0077

[8] Tosco, T., Tiraferri, A. and Sethi, R. (2009) Ionic Strength Dependent Transport of Microparticles in Saturated Porous Media: Modeling Mobilization and Immobilization Phenomena under Transient Chemical Conditions. Environmental Science \& Technology, 43, 4425-4431. http://dx.doi.org/10.1021/es900245d

[9] Tosco, T. and Sethi, R. (2009) MNM1D: A Numerical Code for Colloid Transport in Porous Media: Implementation and Validation. American Journal of Environmental Sciences, 5, 517-525. http://dx.doi.org/10.3844/ajessp.2009.517.525

[10] Amao, A. M. (n.d.) Mathematical Model for Darcy Forchheimer Flow with Applications to Well Performance Analysis. Unpublished Master's Thesis, Texas Tech Univeristy, Lubbock.

[11] Fourie, F.D. (2007) Limitations of the Helmholtz-Smoluchowski Equation in Electroseismics. 10th SAGA Biennial Technical Meeting and Exhibition, Wild Coast Sun, Port Edward, KwaZulu-Natal, 15-18 October 2007.

[12] Alley, W.M., Ed. (1993) Regional Ground-Water Quality. Van Nostrand Reinhold, New York.

[13] Cameselle, C., Reddy, K.R., Darko-Kagya, K. and Khodadoust, A. (2013) Effect of Dispersant on Transport of Na- 
noscale Iron Particles in Soils: Zeta Potential Measurements and Column Experiments. Journal of Environmental Engineering, 139, 23-33. http://dx.doi.org/10.1061/(ASCE)EE.1943-7870.0000473

[14] Malama, B. (2012) Estimation of the Electrokinetic Coupling Coefficient and Hydraulic Conductivity from Streaming Potential Measurements in a Falling-Head Permeameter. NGWA Ground Water Summit, Garden Grove, 6-10 May 2012.

[15] Naudet, V., Revil, A., Rizzo, E., Bottero, J.Y. and Bégassat, P. (2004) Groundwater Redox Conditions and Conductivity in a Contaminant Plume from Geoelectrical Investigations. Hydrology and Earth System Sciences, 8, 8-22. http://dx.doi.org/10.5194/hess-8-8-2004

[16] Engler, T. W. (2010) Part of Collection of Notes from the Petroleum Engineering 524 Class at New Mexico Tech.

[17] Wallace, S.H., Shaw, S., Morris, K., Small, J.S., Fuller, A.J. and Burke, I.T. (2012) Effect of Groundwater pH and Ionic Strength on Strontium Sorption in Aquifer Sediments: Implications for ${ }^{90} \mathrm{Sr}$ Mobility at Contaminated Nuclear Sites. Applied Geochemistry, 27, 1482-1491. http://dx.doi.org/10.1016/j.apgeochem.2012.04.007

[18] Krajangpan, S., Chisholm, B., Kalita, H. and Bezbaruah, A. (2009) Challenges in Groundwater Remediation with Iron Nanoparticles: Enabling Colloidal Stability. Nanotechnologies for Water Environment Applications, 191-212. http://dx.doi.org/10.1061/9780784410301.ch08 